\title{
Importance of covariance in confirmatory factor analysis: safety attitudes questionnaire-chinese version as an example
}

The general look of Figure 1 is quite familiar to us: Yes, it is a typical confirmatory factor analysis (CFA) model. However, having it feel familiar and understanding it thoroughly are completely different. CFA is one of the fields that allows for such discrepancy. This editorial aims to close the gap.

To make the explanation as simple as possible, we use the Safety Attitudes Questionnaire-Chinese version (SAQ-C) as an example, trying to measure the construct of safety culture. SAQ-C has five domains: Teamwork Climate (TW), Safety Climate (SC), Job Satisfaction (JS), Perception of Management (PM), and Working Condition (WC). ${ }^{1}$ Though each domain of the original SAQ-C contains a few items, we assume there are only two for each domain, although this is statistically unrealistic. Figure 1 is a typical CFA model with respect to SAQ-C, the model that is also called a correlated factor model. $^{2}$

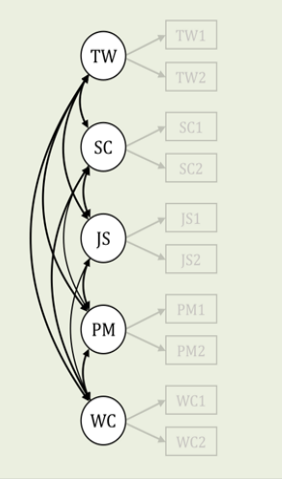

Figure I A five-domain confirmatory factor model (e.g., SAQ-C).

Now, please ruminate about what we usually do with a survey questionnaire. First, we develop a measurement instrument, then a pilot study on a small population follows. The data from the pilot study are finally plugged into a model, which means that the collected data are fed into rectangles in the figure as item responses. Once we reach this point, we run a CFA. Several numbers are reported on the screen, among which we are focused on model fit indices, such as GFI, RMSEA, AGFI, TLI, and so forth. Once those values are determined to be satisfactory, finally, it is time to make the biggest mistake: removing all the tangle of double-headed arrows on the left side, leaving domain-level information only (circles and rectangles at a low opacity). From now, each domain is independent; in Figure 1, we can calculate TW score (circle in which TW is inside), likely by calculating the mean score of two items, TW1 and TW2. The same rule applies to all the other domains.

To a prudent person, this does not make much sense. She may review what the components of Figure 1 mean: As we all know by now, rectangles stand for items and circles for domains (latent traits).

\author{
Volume 6 Issue 2 - 2017
}

\author{
Heon-Jae Jeong,' Wui-Chiang Lee, ${ }^{2}$ Hsun- \\ Hsiang Liao ${ }^{3}$ \\ 'The Care Quality Research Group, Chuncheon, Korea \\ ${ }^{2}$ Department of Medical Affairs and Planning, Taipei Veterans \\ General Hospital \& National Yang-Ming University School of \\ Medicine, Taipei, Taiwan \\ ${ }^{3}$ Deputy Executive Officer, Joint Commission of Taiwan, Taiwan
}

Correspondence: Wui-Chiang Lee, Department of Medical Affairs and Planning, Taipei Veterans General Hospital \& National Yang-Ming University School of Medicine, Taipei, Taiwan, Tel +886-228757| 20, Fax +886-2-28757200, Email leewuichiang@gmail.com

Received: July 12, 2017 | Published: July 20, 2017

Single-headed arrows are factor loadings (just think of it as value for an item's importance). Many people mistakenly call the above ingredients a measurement model. ${ }^{3}$ Although strictly speaking that is not true, let us leave that way. That is not today's main dish, and that is why they are depicted at a low opacity level.

The starter is over. Now, think about the tangle of solid doubleheaded arrows on the left side, which is destined to be forgotten within a few minutes after satisfactory CFA. If they had been so worthless, why should they have been in the CFA? The answer is that they are extremely important and represent covariance among domains (i.e., each double-headed arrow connects two circles). ${ }^{4}$ Covariance means that once something changes, a certain amount of change occurs in the other thing. Getting complicated? No, it should not be; follow us.

Let us give a very intuitive- hypothetical, but realistic - example with SAQ-C domains (refer to Figure 1.). If people's response for one of the two items in the working climate (WC) domain improves, we usually just report that the working conditions got better. However, can this be the end of the story? No, probably having better working conditions leads to better job satisfaction, which may influence the teamwork climate and the safety climate. Only if we look down along this kind of chain reaction can we understand the relationship among domains and their relationships, which are represented by circles and the double-headed arrows, respectively. That whole picture then can eventually describe the construct and concept we want to investigate thoroughly (i.e., safety culture in SAQ-C).

The point is, in CFA, what we prove with the model is not that a certain domain explains a few, the less opaque part of the model. What we really should understand is the covariance, double-headed arrows and their values.

We have zero intention to negate the works you have done so far. In many cases, such covariance might be negligible, or the practical goal 
of a project can be reached without considering variance-covariance structure. Through this article, our only hope is to emphasize that those arrows are important and, therefore, should never be dispensed with after the CFA model is run, as candles decorating a cake are thrown out immediately after a party.

Actually, it is a researcher's call whether to utilize all this information and pursue more comprehensive estimates or not. However, if your hesitation arises from seemingly difficult calculations, rest assured. Most statistical packages allow us to get the model-based results with only one or two clicks. You do not need to decide right now whether to leave your safety zone for a new world. Even so, we can guarantee that the safety zone will not be safe enough really soon; computing power increases too fast.

By the way, to help you understand the covariance tangle, we created a brand-new way to detangle them (Figure 2). It contains the exact same information that Figure 1 has, just in a much easier format. Bon appetite, and welcome the brave new world!



Figure 2 A five-domain confirmatory factor model (SAQ-C): transformed from Figure I.

\section{Acknowledgements}

None.

\section{Conflicts of interest}

None.

\section{References}

1. Lee WC, Wung HY, Liao HH, et al. Hospital safety culture in Taiwan: a nationwide survey using Chinese version Safety Attitude Questionnaire. BMC Health Serv Res. 2010;10(1):234.

2. Jeong HJ, Lee WC. Item Response Theory-Based Evaluation of Psychometric Properties of the Safety Attitudes Questionnaire-Korean Version (SAQ-K). Biom Biostat Int J. 2016;3(5):00079.

3. Acock AC. Discovering structural equation modeling using Stata. Stata Press Books. 2013;p. 51.

4. Hooper DJ, Coughlan J, Mullen M. Structural equation modelling: Guidelines for determining model fit. 2008;p. 2. 